\title{
Performances zootechniques et pratiques des éleveurs en élevage bovin naisseur à la Réunion
}

\author{
J.P. Choisis ${ }^{1,2 *}$ C. Lassalle ${ }^{1}$ \\ S. Messad ${ }^{3}$ P. Grimaud 1,3
}

Mots-clés

Bovin - Vache allaitante - Conduite d'élevage - Performance animale Analyse multivariée - Réunion.

\begin{abstract}
Résumé
A la Réunion, I'insularité et la petite dimension des exploitations font que I'amélioration de la productivité dans les élevages bovins passe par un accroissement de la maîtrise technique. Afin d'analyser les relations entre les pratiques de conduite et les performances animales, dix élevages bovins naisseurs situés dans les Hauts de la Réunion ont fait l'objet d'un suivi de 1999 à 2002. Les données collectées ont été organisées en trois tableaux composés respectivement de 4,8 et 3 variables : les performances animales (intervalle vêlage-vêlage, taux de mise bas, poids à âge type, production bovine de viande vive), les pratiques (temps de présence à l'hectare et par parcelle, temps d'interpassage, taille des parcelles, chargement, complémentation des broutards et des vaches, taux de réforme) et le milieu (pluviométrie, volume d'herbe, état corporel des vaches). Les deux premiers tableaux ont été soumis à une analyse de co-inertie afin d'analyser les relations entre performances zootechniques et pratiques des éleveurs. Les deux tableaux étaient significativement corrélés et les résultats de l'analyse de co-inertie ont été interprétés à l'échelle de chaque exploitation. Au-delà de contraintes spécifiques, ils ont révélé des proximités entre exploitations et entre modes de conduite fondés sur des stratégies variées en cohérence avec les performances observées. Une analyse Statico a été réalisée afin d'évaluer la liaison entre les paramètres de performances et ceux du milieu pour les quatre années étudiées. Elle a montré qu'il existait une costructure stable entre les tableaux milieu et performances, suggérant un effet très structurant des pratiques sur les performances et l'existence de régulations du système qui modèrent les effets du climat.
\end{abstract}

\section{INTRODUCTION}

A la Réunion, l'élevage bovin « conventionnel» est une activité récente. La filière allaitante s'est structurée en quelques décennies selon un modèle biogéographique original : les troupeaux naisseurs se sont installés dans les Hauts de l'île où les animaux sont conduits au pâturage, tandis que les broutards issus de ces élevages sont vendus vers l'âge de dix mois à des ateliers d'engraissement situés dans les Bas où ils sont finis à l'auge. L'élevage naisseur,

1. Cirad, Pôle élevage, St Pierre, F-97410 la Réunion.

2. Inra UMR 1201, Castanet -Tolosan, F-31326 France.

3. Cirad, UPR Systèmes d'élevage, Montpellier, F-34398 France.

Auteur pour la correspondance

* Inra, UMR 1201 Dynafor, Castanet-Tolosan, F-31326 France.

Tél. : +33 (0)561285560; fax : +33 (0)561285411

E-mail : jean-philippe.choisis@toulouse.inra.fr qualifié de professionnel, représente plus de 11000 femelles de plus de huit mois, réparties dans 422 élevages ${ }^{4}$ essentiellement situés dans les Hauts de l'Ouest et à la Plaine des Cafres (figure 1). Avec le développement de la filière, les éleveurs et leurs services d'appui, soucieux d'améliorer les performances zootechniques, se sont beaucoup inspirés des modèles d'élevage métropolitains en important des animaux, de races Limousine et Blonde d'Aquitaine majoritairement, des équipements et des techniques. Ces choix techniques se sont traduits par une évolution notable de la production de viande qui est passée de 800 à 1800 t équivalent carcasse en 30 ans. Toutefois, si les performances de croissance en atelier d'engraissement se sont rapidement améliorées avec la maîtrise des techniques de rationnement, les performances des élevages naisseurs restent très hétérogènes et encore très en deçà de leurs

\footnotetext{
${ }^{4}$ Déclaration auprès du ministère de l'Agriculture et de la Pêche
} 


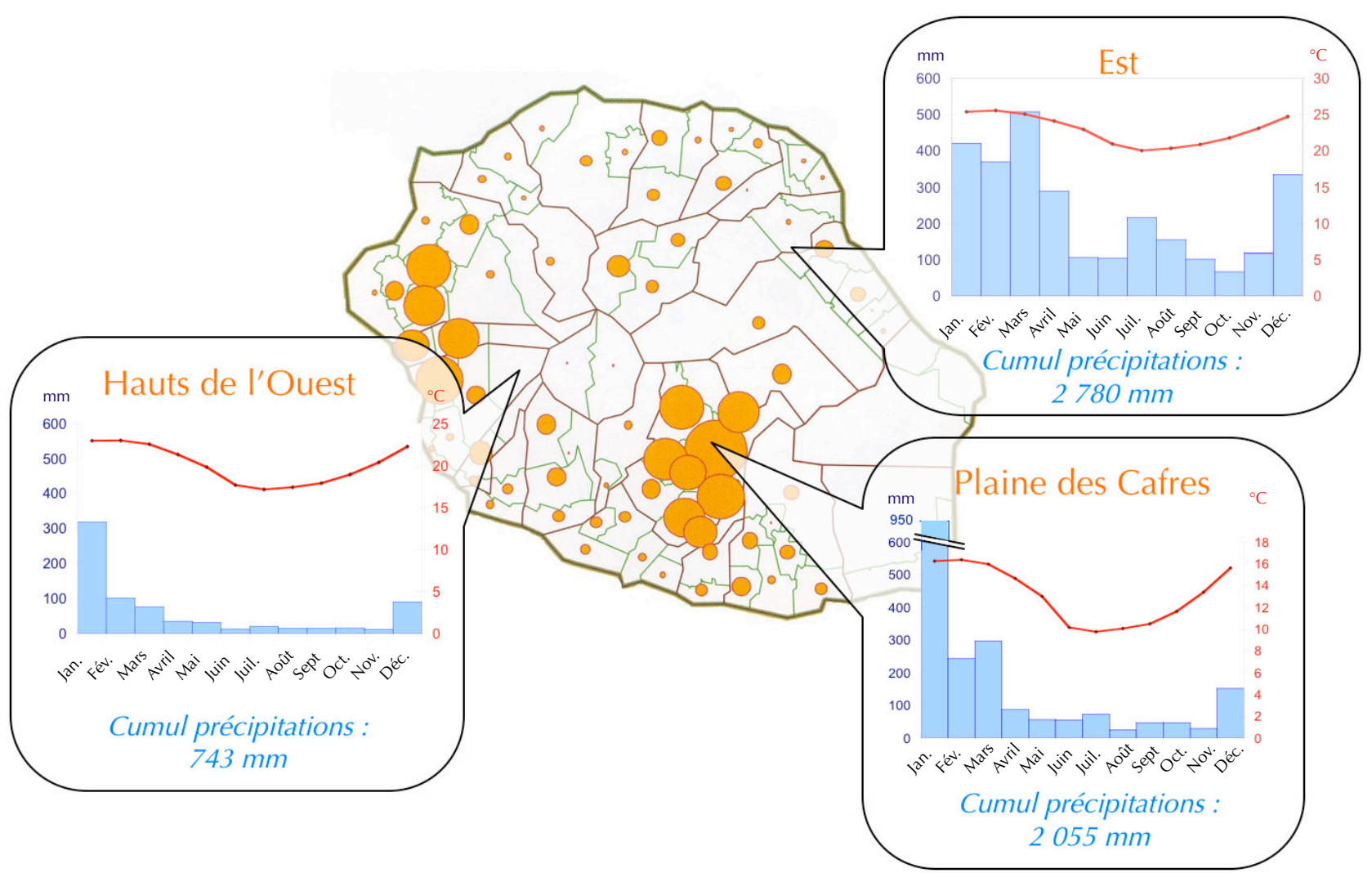

Figure 1 : pluviométrie et température mensuelles pour trois zones géographiques de la Réunion (moyennes calculées sur les années 1999-2002) et carte de répartition des effectif de vaches allaitantes. Source : recensement général agricole, 2000.

homologues métropolitains : l'indice de productivité moyen, calculé à partir de 32 élevages naisseurs en 2001, était proche de $220 \mathrm{~kg}$ de viande vive produite annuellement par vache présente (14), alors que les réseaux d'élevage français atteignent des valeurs moyennes supérieures à $360 \mathrm{~kg}$ par vache (13).

Si les contraintes de milieu particulières de la Réunion peuvent justifier d'une moindre productivité, on observe des différences notables entre exploitations qui laissent augurer des marges de progrès encore importantes. Selon les types d'exploitation, l'indice de productivité a varié entre $180 \mathrm{~kg}$ et $273 \mathrm{~kg}$ avec des variations de 1 à 3 entre exploitations extrêmes (14). Cette hétérogénéité des performances entre exploitations peut être en partie reliée au fait qu'au-delà du matériel animal et des techniques, c'est aussi le métier d'éleveur qui est en cours de construction. De ses choix en termes d'orientation des systèmes techniques et de conseil dépendra l'amélioration des performances économiques de son exploitation. Ainsi, l'agrandissement des exploitations et l'extensification, qui sont les tendances qui prévalent en Europe, ne sont pas socialement acceptables à la Réunion du fait de l'exiguité de l'île et du contexte de chômage élevé (4). L'emploi étant une priorité locale, le maintien des exploitations existantes, voire l'installation de jeunes exploitants sont des objectifs affichés. La marge de manœuvre est donc relativement étroite et l'amélioration de la productivité passe, selon un constat partagé par les acteurs techniques de la filière, par un accroissement de la maîtrise technique et, pour les élevages naisseurs en particulier, par une gestion plus fine de la ressource en herbe. D'où la nécessité de produire des références afin d'adapter le conseil technique aux contraintes spécifiques de la Réunion.

Cet objectif passe par la mise en œuvre de dispositifs de suivi des exploitations. La mesure des performances et l'enregistrement des pratiques des éleveurs sont censés permettre l'identification des
« bonnes pratiques », c'est-à-dire celles qui conduisent à l'expression des meilleurs résultats dans un champ de contraintes donné. Une finalité souvent exprimée est de tirer des enseignements des élevages les plus performants. Ces bonnes pratiques ne sont toutefois pas simples à identifier, car, au-delà des contraintes spécifiques (climatiques, topographiques, etc.) qui sont souvent mises en avant par les éleveurs, ce sont aussi les stratégies personnelles qui les conditionnent (réduire les dépenses, maximiser les primes, travailler à l'extérieur, dégager du temps libre, etc.). La diversité des contextes de production et des stratégies rend toute généralisation des résultats très délicate et soulève le problème des critères d'évaluation des performances de l'exploitation. Ces critères doivent permettre d'appréhender l'ensemble des stratégies mises en œuvre par les éleveurs (priorité à l'utilisation des surfaces, aux performances individuelles...).

L'objectif de la présente étude a été de faire le lien entre les pratiques de conduite de troupeaux bovins allaitants, en matière de gestion du pâturage en particulier, et les performances zootechniques obtenues.

\section{MATERIEL ET METHODES}

\section{Contexte de suivi des exploitations}

Un suivi des pratiques et des performances animales a été mis en œuvre, de 1999 à 2002, sur dix élevages naisseurs situés dans les Hauts de la Réunion. Il s'est inscrit dans la continuité des travaux de recherche menés au début des années 1990 sur l'agroécologie des pâturages à la Réunion (1), selon un dispositif résultant d'une collaboration entre l'Union des associations foncières pastorales (Uafp), l'Etablissement départemental de l'élevage (EDE), la société coopérative Sica Réunion viande (SicaRévia) et le Pôle 
élevage du Cirad à la Réunion (21). L'objectif était d'analyser les performances des troupeaux au regard des pratiques et des stratégies développées par les éleveurs. Le suivi s'est fondé sur un passage trimestriel dans les exploitations afin d'effectuer les mesures et prélèvements sur les animaux (pesée des broutards, notation d'état corporel et prises de sang sur les vaches) et sur les parcelles (hauteurs d'herbe). Ces données ont été complétées par les inventaires et les mouvements d'animaux fournis par l'EDE, et par des entretiens avec les éleveurs sur les pratiques mises en œuvre. Chaque éleveur avait également à sa charge de remplir un calendrier de pâturage contenant les informations sur la fertilisation des prairies, le rythme et l'ordre de rotation sur les parcelles et la complémentation distribuée par lot d'animaux.

Parmi les dix éleveurs suivis, cinq étaient situés à la Plaine des Cafres, quatre dans les Hauts de l'Ouest et un dans l'Est. Dans l'Est la pluviométrie très importante entraîne des problèmes de parasitisme et de dégradation rapide des prairies. En revanche, les deux autres régions sont confrontées à une saisonnalité marquée de la pluviométrie : l'hiver se caractérise par une sécheresse qui est plus prononcée dans les Hauts de l'Ouest, où la pousse de l'herbe en saison fraîche sèche (SS) est réduite, tandis que la pluie tombe en abondance pendant la saison humide (SH), surtout au mois de janvier (figure 1).

Les exploitations suivies ont été sélectionnées parmi les élevages naisseurs au pâturage mettant en œuvre des pratiques de fertilisation et de rotation de prairies, et inscrits dans des réseaux techniques. En conséquence, ils n'étaient pas représentatifs de la diversité des systèmes d'élevage à la Réunion et leurs performances étaient globalement supérieures à la moyenne des élevages adhérents à la SicaRévia. L'échantillon ne comptait que des exploitations de taille moyenne à grande, avec une surface fourragère principale comprise entre 25 et 75 ha et un nombre de vaches allaitantes compris entre 42 et 69 . Le chargement moyen a varié de 0,8 à $2,5 \mathrm{UGB} / \mathrm{ha}$ (tableau I).

\section{Données collectées}

Les données recueillies au cours du suivi ont été classées en trois catégories. Le premier jeu de données était constitué par les performances zootechniques des exploitations, individuelles [gains moyens quotidiens (Gmq), poids à âge type (Pat), intervalle vêlage-vêlage (Ivv)] et globales [taux de mise bas, taux de mortalité, production bovine de viande vive (Pbvv)]. Ce dernier paramètre, utilisé dans les réseaux d'élevage français, a permis de disposer d'une vision synthétique des performances de l'exploitation (18). La Pbvv correspondait, dans la présente étude, à la production de l'année de viande de broutards mâles et femelles sur pied, estimée à partir des courbes de croissance. Un deuxième jeu de données a concerné les pratiques des éleveurs : temps de présence des animaux et temps de repos des parcelles, chargement à l'hectare, complémentation des animaux, fertilisation des parcelles, taux de réforme du troupeau. Enfin, pour le troisième, certaines variables indicatrices de l'état du système ont été relevées : hauteurs et biovolumes d'herbe par saison, notes d'état corporel des vaches allaitantes, pluviométrie.

\section{Analyses statistiques}

L'exploitation statistique des données s'est faite d'après un choix des variables basé sur des critères de pertinence, de non-redondance (Gmq et Pat, par exemple, fournissent la même information) et d'exhaustivité des données. Certaines informations incomplètes, comme la fertilisation, n'ont ainsi pas été retenues. Ces différentes variables sont présentées dans le tableau II. Les associations entre performances zootechniques et pratiques d'élevage ont été étudiées par la méthode d'analyse de co-inertie (6). Les tableaux des performances et des pratiques ont été pris en compte par la co-inertie de manière symétrique, l'un n'étant pas considéré explicatif de l'autre. Cela est compensé par la résistance de la méthode aux conditions de colinéarité entre variables et de nombre d'individus nécessairement important dans le cadre des analyses à couplage dissymétrique où un tableau est considéré explicitement comme prédicteur de l'autre (3). Le coefficient RV de corrélation vectorielle (16) et le test de signification de Monte-Carlo basé sur des permutations aléatoires des individus ont permis de juger de la liaison globale entre les deux tableaux de paramètres. Par ailleurs, les conditions climatiques, qui apparaissaient extrêmement variables entre années, ont souvent été mises en avant par les éleveurs pour justifier de difficultés particulières. Une analyse complémentaire a donc été conduite pour apprécier la stabilité temporelle du lien probable entre les performances des exploitations et le milieu selon la méthode Statico (19). Elle a fourni une représentation « compromis », appelée également costructure moyenne, entre les différentes années. Ces analyses ont été réalisées à l'aide de Ade4 Package $(2,7,20)$ sous l'environnement $\mathrm{R}(15)$.

Tableau I

Principales caractéristiques des dix élevages suivis

\begin{tabular}{lllcccc} 
Eleveur & Localisation & Activité & SFP (ha) & Nb. de vaches & Nb. d'UGB & Chargement (UGB/ha) \\
\hline AR & Est & Naisseur - engraisseur & 37 & 62 & 91 & 2,46 \\
BE & Plaine des Cafres & Naisseur - engraisseur & 72 & 62 & 87 & 1,21 \\
BO & Hauts de l'Ouest & Naisseur & 60 & 55 & 60 & 1,01 \\
CO & Plaine des Cafres & Naisseur & 38 & 48 & 59 & 1,55 \\
FO & Hauts de l'Ouest & Naisseur & 25 & 53 & 64 & 2,55 \\
GA & Hauts de l'Ouest & Naisseur & 29 & 45 & 50 & 1,72 \\
HO & Hauts de l'Ouest & Naisseur & 75 & 52 & 64 & 0,85 \\
OD & Plaine des Cafres & Naisseur & 66 & 42 & 52 & 0,79 \\
PA & Plaine des Cafres & Naisseur & 55 & 69 & 82 & 1,49 \\
PI & Plaine des Cafres & Naisseur - sélectionneur & 60 & 53 & 62 & 1,05
\end{tabular}




\section{Tableau II}

Variables retenues pour l'analyse de co-inertie et valeurs moyennes et dispersion des indicateurs de performances et de pratiques

\begin{tabular}{|c|c|c|c|c|c|c|c|}
\hline Tableau & Variables & & Moy. & Min. & Max. & Ecart-type & CV $(\%)$ \\
\hline \multirow[t]{4}{*}{ Performances } & Intervalle vêlage-vêlage & $\operatorname{lvv}(j)$ & 374,6 & 351,7 & 423,7 & 26,7 & 7,1 \\
\hline & Taux de mise bas & $\mathrm{Mb}(\%)$ & 80,6 & 58,3 & 94,8 & 13,0 & 16,1 \\
\hline & $\begin{array}{l}\text { Poids à âge type } 120 \text { jours } \\
\text { pour les mâles }\end{array}$ & P120m (kg) & 149,0 & 121,2 & 180,1 & 21,5 & 14,4 \\
\hline & $\begin{array}{l}\text { Production bovine de viande } \\
\text { vive ramenée à l'ha } \\
\text { de surface fourragère }\end{array}$ & $\mathrm{Pbvv/ha}(\mathrm{kg})$ & 274,8 & 150,3 & 397,9 & 92,0 & 33,5 \\
\hline \multirow[t]{8}{*}{ Pratiques } & $\begin{array}{l}\text { Nb. moyen de jours } \\
\text { de présence à l'ha }\end{array}$ & $J p(j)$ & 8,0 & 0,8 & 29,4 & 8,8 & 109,9 \\
\hline & Taille moyenne des parcelles & Parc (ha) & 2,8 & 1,1 & 5,4 & 1,8 & 64,1 \\
\hline & $\begin{array}{l}\text { Temps d'interpassage moyen } \\
\text { de la rotation }\end{array}$ & $\mathrm{Ti}(\mathrm{j})$ & 74,5 & 37,5 & 148,0 & 35,9 & 48,2 \\
\hline & $\begin{array}{l}\text { Temps de présence moyen } \\
\text { des animaux sur les parcelles }\end{array}$ & $\operatorname{Tp}(j)$ & 15,6 & 2,7 & 49,0 & 14,9 & 95,5 \\
\hline & Chargement surface fourragère & UGB (UGB/ha) & 1,5 & 0,8 & 2,6 & 0,6 & 42,6 \\
\hline & Complémentation des broutards & Cbrou (UF/an) & 434,2 & 0,0 & 857,5 & 270,0 & 62,2 \\
\hline & $\begin{array}{l}\text { Complémentation des vaches } \\
\text { allaitantes }\end{array}$ & Cva (UF/an) & 741,9 & 46,0 & 1527,1 & 429,7 & 57,9 \\
\hline & Taux de réforme & $\operatorname{Ref}(\%)$ & 11,3 & 4,7 & 17,7 & 4,4 & 39,4 \\
\hline
\end{tabular}

RESULTATS

\section{Indicateurs de performances et de pratiques des éleveurs dans les exploitations}

Les valeurs des différentes variables apparaissent dans le tableau II, où chaque exploitation est représentée par sa valeur moyenne sur les années du suivi. Malgré une certaine homogénéité de l'échantillon, les performances zootechniques tout comme les critères d'appréciation des pratiques ont montré une grande dispersion.

\section{Relations entre performances zootechniques et pratiques d'élevage}

L'analyse de co-inertie a montré une corrélation significative entre le tableau des performances zootechniques et celui des pratiques des éleveurs (coefficient RV de 0,52; test de permutation de Monte-Carlo significatif). Les deux premiers axes de cette analyse représentaient respectivement 80,7 et 18,4 p. 100 de la co-inertie. La projection des individus sur le plan 1-2 (figure 2) a montré, d'une part, une relative superposition entre les projections dérivées des pratiques et des performances et, d'autre part, des proximités entre exploitations, suggérant des caractéristiques proches. La projection des variables de performances (figure 3a) a permis de préciser que l'axe 1 était fortement lié aux performances individuelles (Ivv, taux de mise bas et $\mathrm{P} 120 \mathrm{~m}$ ), alors que la production globale à l'hectare $(\mathrm{Pbvv} / \mathrm{ha})$ discriminait l'axe 2. La lecture du plan composé par les variables de pratiques (figure $3 \mathrm{~b}$ ) a montré le poids des variables qui mesuraient l'utilisation dans le temps de ces parcelles (Jp, Ti, Tp), alors que la taille de ces parcelles (Parc) intervenait peu. Le chargement (UGB) et la complémentation des

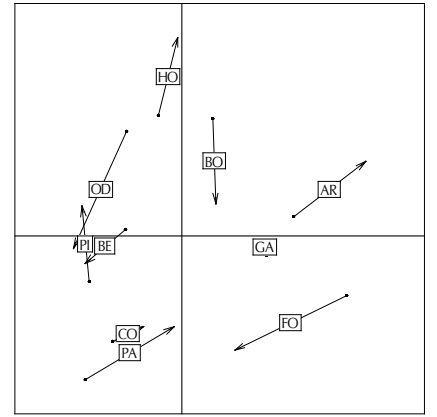

Figure 2 : projection des exploitations sur le plan 1-2 de l'analyse de co-inertie. L'origine et la pointe de la flèche représentent respectivement leur position vis-àvis des performances et des pratiques. La longueur du segment est proportionnelle à l'adéquation des deux thèmes pour décrire l'exploitation. vaches (Cva) ont fortement contribué, dans le même sens, à l'axe 2 (figure $3 \mathrm{~b}$ ), alors que les pratiques de complémentation des vaches et des broutards n'ont pas semblé reliées. Enfin, le rapprochement des deux projections (figures $3 a$ et $3 b$ ) a mis en évidence les relations qui existaient entre (i) chargement / complémentation des vaches et productivité globale, (ii) complémentation des broutards et leurs performances de croissance, (iii) âge de réforme et performances individuelles, et (iv) rythme de rotation des parcelles et performances zootechniques, individuelles et globales.

\section{Analyse individuelle des exploitations}

La petite dimension de l'échantillon a permis une analyse individuelle des exploitations à partir de leur projection sur le plan de co-inertie (figure 2). Les six groupes suivants ont été distingués :

- éleveurs $\mathrm{CO}$ et PA, qui ont obtenu de bonnes performances individuelles (Pat à 120 jours de l'ordre de $170 \mathrm{~kg}$ et Ivv de 354 jours) 

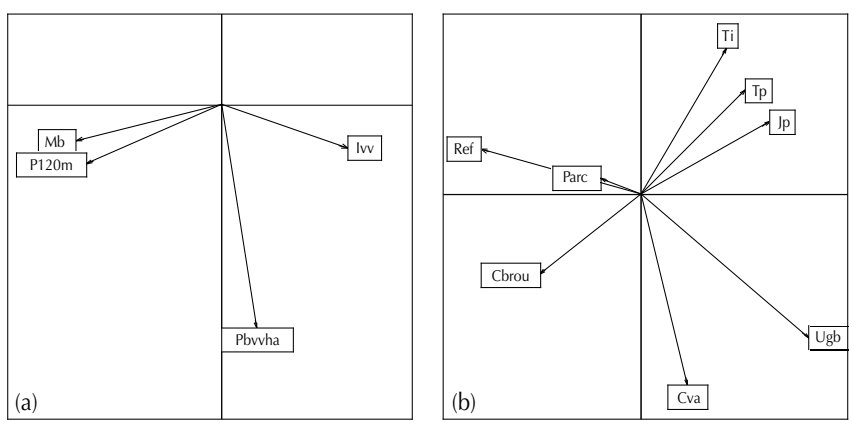

et globales (375-398 kg/ha). Dans ces deux exploitations, le chargement moyen a été de 1,5 UGB/ha et les broutards et les vaches ont été complémentés de manière très soutenue. Leur système de rotation était rapide (temps d'interpassage de 45 à $60 \mathrm{j}$ ) avec des temps de présence assez courts (6-10 j). Il en a résulté des volumes d'herbe disponibles (Vhd) par UGB identiques : environ $700 \mathrm{~m}^{3}$ en $\mathrm{SS}$ et $970 \mathrm{~m}^{3}$ en SH, et une note d'état corporel à la mise bas supérieure ou égale à 2 ;

- éleveurs BE et PI, assez proches des précédents au niveau des performances individuelles. Leur gestion de la ressource fourragère et de la complémentation des broutards a été similaire, mais les parcelles ont été de plus grande taille (environ 5 ha vs 1,5 à 2 ha pour les précédents) et la durée de présence moyenne par hectare a donc été plus faible (de 0,8 à 1,6 j). Ils ont présenté de moins bonnes performances à l'hectare $(260-289 \mathrm{~kg} / \mathrm{ha})$, en raison d'un chargement plus faible (1 à 1,2 UGB/ha) et d'une complémentation moins forte des vaches. Avec un chargement de $1 \mathrm{UGB} / \mathrm{ha}$, le disponible fourrager de PI a été élevé et les Vhd importants ont peu varié entre les saisons (1 359 vs $\left.1255 \mathrm{~m}^{3}\right)$. Cette abondance fourragère s'est traduite par la note d'état corporel la plus élevée $(2,4)$ pour ses vaches qui n'ont reçu qu'une complémentation minimale. C'est également l'éleveur qui a eu les performances de croissance les plus élevées. Sa logique économique visait à faire exprimer le maximum du potentiel de croissance car il vendait des animaux reproducteurs à plus haute valeur ajoutée ;

- éleveur OD ; il se situait également dans la fourchette supérieure des performances individuelles mais sa productivité globale a été beaucoup plus faible $(162 \mathrm{~kg} / \mathrm{ha})$ du fait d'une conduite très extensive $(0,8 \mathrm{UGB} / \mathrm{ha})$. En revanche, ses pratiques ont été proches des précédentes avec un rythme de rotation rapide [temps de présence moyen des animaux sur les parcelles (Tp) de 2,7 j] et une complémentation des broutards élevée. Le faible chargement et la petite dimension des parcelles (1 ha) ont fait que les temps d'interpassage (Ti) étaient longs (67 j). Comme pour les quatre éleveurs précédents, son exploitation était située à la Plaine des Cafres ;

- éleveurs HO et BO ; les performances individuelles moyennes, combinées à un faible chargement $(0,8$ à $1 \mathrm{UGB} / \mathrm{ha})$ ont abouti aux plus faibles Pbvv. $\mathrm{HO}$ avait une conduite simplifiée des lots avec des temps de présence très longs $(34 \mathrm{j})$ sur de grandes parcelles (> 5 ha). Cela a abouti à des Ti très longs (148 j). C'était le seul éleveur qui ne complémentait pas ses broutards, ce qu'il justifiait par une forte disponibilité en herbe $\left(2400 \mathrm{~m}^{3}\right.$ en SH et $1800 \mathrm{~m}^{3}$ en SS). BO avait un rythme de rotation intermédiaire entre HO et les éleveurs de la Plaine des Cafres (Tp de 16 j). Il complémentait les vaches et les broutards mais à un niveau inférieur aux éleveurs de cette zone. Sa gestion extensive le situait à des niveaux de disponibilité de l'herbe élevés (1 $200 \mathrm{~m}^{3}$ en SH et $900 \mathrm{~m}^{3}$ en SS) ;

- éleveur GA ; il a présenté des performances individuelles identiques à $\mathrm{BO}$, mais un chargement distinct a empêché qu'ils figurent à proximité sur le tableau des performances : celui de GA était
Figure 3 : projection des variables de performances (a) et de pratiques (b) sur le plan de co-inertie 1-2. Mb : taux de mise bas ; P120m : poids à âge type 120 jours pour les mâles; Ivv : intervalle vêlage-vêlage; Pbvv/ha : production bovine de viande vive ramenée à l'ha de surface fourragère Ref : taux de réforme; Parc : taille moyenne des parcelles; $\mathrm{Ti}$ : temps d'interpassage moyen de la rotation; Tp : temps de présence moyen des animaux sur les parcelles ; Ip : nb. moyen de jours de présence à l'ha ; Cbrou / Cva : complémentation des broutards / des vaches allaitantes; Ugb : chargement en Ugb/ha de surface fourragère.

beaucoup plus élevé (1,7 vs $1 \mathrm{UGB} / \mathrm{ha})$, conduisant à une productivité à l'hectare plus élevée (291 kg/ha). Ils ont été, en revanche, proches sur le tableau des pratiques, en raison d'une conduite des lots similaire (Tp de $16 \mathrm{j}$ ), avec des parcelles de taille peu différente (1 vs 1,6 ha), d'où des Jp assez élevés (11-15 j) et des niveaux de complémentation voisins. Cependant, le chargement plus élevé chez GA s'est traduit par des Vhd beaucoup plus réduits (environ $\left.400 \mathrm{~m}^{3} / \mathrm{UGB}\right)$;

- éleveurs AR et FO ; ils ont eu les performances individuelles les plus faibles (taux de mise bas et P120m de l'ordre de 0,6 et $120 \mathrm{~kg}$ ). A contrario, ils ont eu le chargement le plus élevé (2,5 UGB/ha), ce qui leur a permis de compenser la faible productivité individuelle et d'atteindre une assez bonne performance globale (300-365 kg/ha). Ce fort chargement a conduit à d'assez faibles disponibilités fourragères $\left(500-685 \mathrm{~m}^{3} / \mathrm{UGB}\right)$ bien qu'elles aient été supérieures à celles de GA avec des différences marquées entre saisons (bien moindre disponibilité en SS). La complémentation des vaches a été assez élevée afin de compenser partiellement cette moindre disponibilité. Le déficit fourrager s'est toutefois répercuté sur l'état des vaches (note d'état corporel la plus faible : 1,6-1,7) et sur les performances individuelles. Ces deux exploitations, assez proches dans la logique, et les performances étaient éloignées sur le plan des pratiques du fait d'une différence de gestion de la ressource, exprimée par des rythmes de rotation différents (49 vs $9 \mathrm{j}$, respectivement chez AR et FO).

\section{Effet de l'année}

Le test de Monte-Carlo, basé sur le critère d'inertie interannées, a montré qu'il existait un effet significatif de l'année sur le milieu $(p=0,014)$ mais pas sur les performances $(p=0,6)$. Les scores des tableaux - années, issus de l'analyse Statico, ont indiqué que toutes les années avaient participé de manière égale au compromis (figure 4). Il existait une costructure stable entre les tableaux milieu et performances, en particulier pour les années 1999, 2001 et 2002, projetées à proximité. La projection des individus sur le plan 1-2 pour les différentes années (figure 5) confirmait la stabilité de la costructure.

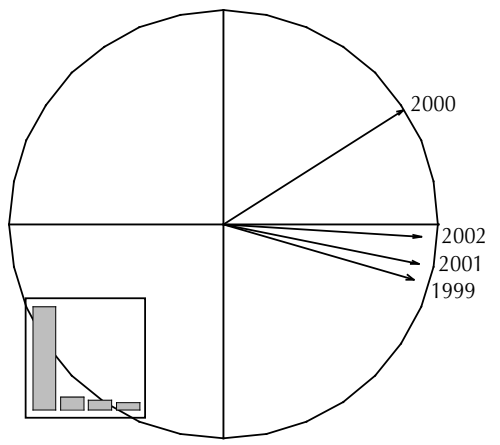

Figure 4 : scores des tableaux - années sur le plan 1-2 de l'analyse Statico qui réalise la synthèse de l'évolution du lien entre performances et milieu. En vignette, le diagramme des valeurs propres de I'analyse Statico. 


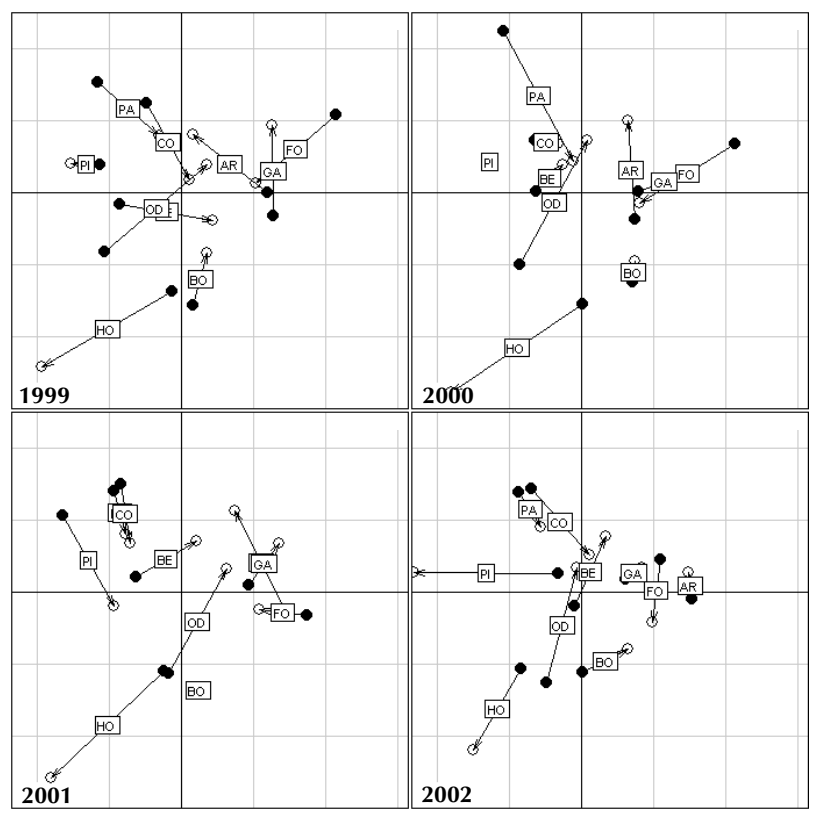

Figure 5 : représentation sur le plan 1-2 de l'analyse Statico de la relation performances (point noir) et milieu (point blanc) entre 1999 et 2002 par exploitation.

\section{- DISCUSSION}

Si le postulat fondateur de ces travaux, c'est-à-dire identifier les bonnes pratiques d'élevage, apparaît pertinent, en particulier aux yeux des conseillers, il comporte un risque implicite. Celui d'associer bonnes pratiques et performances zootechniques élevées. L'échantillon de l'étude, malgré sa petite taille, présentait une hétérogénéité des résultats techniques. Cette hétérogénéité n'était pas seulement liée aux conditions de milieu spécifiques mais aussi et surtout aux stratégies et pratiques mises en œuvre par les éleveurs.

Cette variabilité des pratiques attachées à la prairie et des systèmes qui leur sont liés est communément rencontrée dans les pays du Nord (22) comme dans ceux du Sud (12). A l'exemple de ces auteurs, les typologies réalisées conduisent à identifier différents niveaux d'intensification allant classiquement de systèmes dits traditionnels à intensifs. Il en était de même à la Réunion, où l'analyse fonctionnelle des systèmes d'alimentation a permis d'identifier différents niveaux d'intensification et de chargement (11).

Face à cette diversité, il n'existe pas de bonnes pratiques génériques et les conseils doivent être adaptés aux situations. C'est pour répondre à cette difficulté que Vivier (22) a proposé le concept de «paquet technique » qui recouvre un ensemble de bonnes pratiques adaptées à chaque système identifié.

Les éleveurs du présent suivi apportent des éléments de justification variés aux conduites et aux niveaux de chargement adoptés. Il s'agit de considérations liées (i) au climat et à la qualité de l'herbe (problème de piétinement et de dégradation de la prairie, chargement limité par la pousse de l'herbe...), (ii) aux coûts (accroître l'autonomie fourragère...) et (iii) au travail (simplifier la conduite...). Moins mise en avant, la valorisation économique de la production semble prépondérante. Elle apporte des éléments de compréhension aux pratiques des éleveurs précédemment décrites. Ainsi, la vente d'animaux reproducteurs à plus forte valeur ajoutée peut justifier un niveau de chargement plus réduit participant à maximiser les performances de reproduction et de croissance. Cette orientation s'accompagne d'un ensemble de pratiques cohérentes : élevage en race pure, taux de réforme plus élevé, rythme de rotation plus rapide pour exploiter une herbe de meilleure qualité, complémentation élevée des broutards, etc.

A l'opposé, les éleveurs qui produisent des broutards destinés à l'engraissement ont tout intérêt à maximiser leur production globale. Ainsi, la production bovine de viande vive à l'hectare s'accroît avec le chargement, même si les performances zootechniques s'en trouvent affectées. Cette équation n'est toutefois vraie que jusqu'à un seuil de chargement qui dépend de la zone et des pratiques de complémentation. C'est ce que l'on peut percevoir de la relation établie sur les élevages suivis (figure 6). Toutefois, même si un chargement supérieur à 2 UGB/ha ne semble pas souhaitable d'un point de vue technique, il faut également tenir compte du fait que l'augmentation du cheptel de mères s'accompagne d'une augmentation du nombre d'animaux potentiellement primables. Des élevages ayant une moindre performance sur le plan technique peuvent donc être efficaces sur le plan économique.

A l'inverse des précédents éleveurs, ces derniers vont plutôt privilégier la rusticité (croisements) et rechercher des carrières de femelles longues (taux de réforme plus faible). La complémentation des vaches chez ces éleveurs se justifie moins en termes d'équilibre de ration que de tentative de combler le déficit fourrager. La note d'état corporel plus faible mesurée sur les vaches de ces élevages traduit ce phénomène.

Dans la réalité, les éleveurs se positionnent généralement entre ces deux archétypes. Ils fournissent à la fois des animaux destinés à l'engraissement et à la reproduction. Le matériel génétique, le chargement et la complémentation sont les leviers d'action sur lesquels l'éleveur va jouer pour orienter sa production. A partir de leur expérience, les éleveurs évoquent des maxima de chargement à ne pas dépasser.

Par ailleurs, l'argument climatique (mobilisé à travers la zone géographique ou l'année) vient souvent justifier de pratiques ou de performances particulières. Ainsi, les éleveurs des Hauts de l'Ouest, zone où la sécheresse est plus prononcée, mettent souvent en avant le fait qu'il est plus facile de faire de l'élevage à la Plaine des Cafres. L'examen global des performances obtenues (croissance des broutards et taux de mise bas réduits d'un quart, en moyenne, par rapport aux éleveurs de la Plaine des Cafres) semble leur donner raison. Les pratiques mises en œuvre au niveau local résulteraient donc d'une adaptation au milieu. Les éleveurs des Hauts de l'Ouest ont ainsi des rythmes de rotation plus lents (temps de présence moyen des animaux sur les parcelles de 19 jours contre 6 jours pour la Plaine des Cafres). Sans nier la relation au climat et aux conditions de milieu particulières, l'analyse de co-inertie permet d'entrevoir une réalité plus complexe. On observe, en

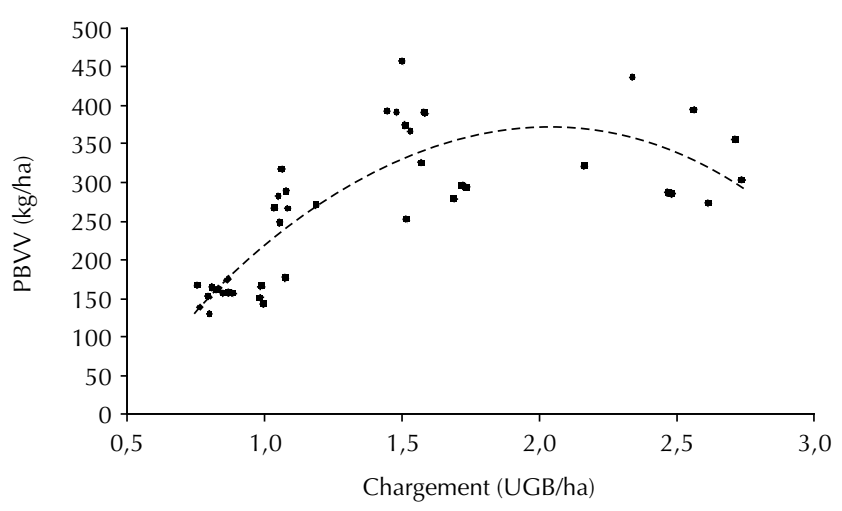

Figure 6 : relation entre chargement et production bovine de viande vive (Pbvv) pour l'ensemble des couples éleveur $x$ année. 
effet, des niveaux de chargement variés dans toutes les zones qui résultent de choix différents de la part des éleveurs. Ces choix se traduisent par des Vhd très variables, indépendamment de la zone géographique. Paradoxalement, c'est chez un éleveur des Hauts de l'Ouest (HO) que l'on rencontre les valeurs les plus élevées.

Des conduites extensives, avec des chargements inférieurs ou égaux à 1 UGB/ha fournissent des Vhd élevés (supérieurs à $1000 \mathrm{~m}^{3} /$ UGB en saison des pluies) et limitent le recours à la complémentation des vaches. Le surdimensionnement de la sole pâturée, dans un souci de simplification de la conduite ou de sécurisation de l'alimentation, est une pratique observée en élevage bovin (8). Associé à un rythme de rotation rapide, c'est-à-dire une faible intensité de pâturage, telle que pratiquée par PI (Tp de 6 j), ce choix permet l'expression de performances zootechniques élevées. A contrario, associé à un rythme de rotation lent, tel que pratiqué par HO (Tp de $34 \mathrm{j}$ ), il conduit à des performances zootechniques faibles (globalement les plus faibles de l'échantillon). Ce phénomène est lié à la moindre qualité de l'herbe offerte qui résulte d'une plus grande proportion de matériel sénescent et d'une moindre digestibilité des feuilles vertes, laquelle décroît avec la longueur de la gaine (8).

Concernant l'influence de l'année, les résultats de l'analyse de la relation milieu - performances ne permettent pas d'identifier un effet particulier de ce facteur. Cette relation est assez stable pour chaque agriculteur. Ceci suggère un effet très structurant des pratiques sur les performances et l'existence de régulations du système (telles que la complémentation ou l'adaptation du rythme de rotation) qui permettent d'amoindrir les effets du climat.

\section{CONCLUSION}

Il existe une littérature abondante, en écologie fonctionnelle, sur le couplage de tableaux. Or, il semble que ces méthodes soient encore peu mises en œuvre dans l'analyse de données d'enquêtes ou de suivi des exploitations agricoles. L'exemple traité ici montre le potentiel de ce type d'analyses pour explorer des jeux de données pour lesquels le nombre élevé de variables mesurées rend l'interprétation difficile. Ces analyses fournissent des représentations graphiques qui permettent une exploration détaillée des données.
Ces méthodes ont ainsi permis aux auteurs de mettre en regard des pratiques et des performances zootechniques et de repérer des groupes d'éleveurs dont les pratiques sont fondées sur des stratégies différentes. Les conseils techniques délivrés aux éleveurs doivent donc nécessairement tenir compte de ces stratégies qui selon les cas vont s'orienter vers la recherche d'une efficience maximale ou, au contraire, vers la sécurisation du système. Pour autant, cela ne signifie pas que toute pratique soit bonne au motif qu'elle est justifiée par une stratégie. Duru et coll. (9) montrent ainsi qu'il existe une gamme de Vhd cohérente avec les objectifs de production. Au-delà d'un certain seuil, augmenter l'intervalle entre deux passages ne fait que réduire la croissance de l'herbe, résultat contradictoire avec la sécurité recherchée.

Les données recueillies dans les élevages suivis montrent aussi que le souci de sécurité et d'autonomie fourragère ne peut justifier un faible chargement. Dans les conditions de la Réunion, en deçà de $1 \mathrm{UGB} / \mathrm{ha}$, il est difficile de maîtriser la pousse de l'herbe. Ce niveau de chargement, associé à un temps de repos des prairies supérieurs à deux mois, conduit à disposer de Vhd très élevés (> $1000 \mathrm{~m}^{3}$ en saison de pluies) et de qualité médiocre, ce qui se traduit par de très faibles performances individuelles et globales.

Certaines proximités dans les pratiques et les performances observées intra zone géographique ne peuvent être uniquement imputées à des considérations pédoclimatiques. Comme l'indique Darré (5), c'est au sein du groupe professionnel local que s'échangent les idées et se construisent les normes. Ces relations vont ainsi conduire à des actions différenciées selon les agriculteurs et les régions. Actions qui sont aussi influencées par la proximité des agriculteurs à l'appareil technique. Les auteurs ont ainsi observé que les conseils formulés sur la gestion des prairies étaient d'autant plus appliqués que le lien avec les conseillers était fréquent et ancien (10).

\section{Remerciements}

Nous tenons à remercier les éleveurs et l'ensemble des agents du Cirad, de l'Uafp, de l'EDE et de la SicaRevia ayant participé à cette opération de recherche.

\section{BIBLIOGRAPHIE}

1. BLANFORT V., 1996. Agro-écologie des pâturages d'altitude à l'île de la Réunion. Pratiques d'éleveurs et durabilité des ressources herbagères dans un milieu à fortes contraintes. Thèse Doct., Université Paris-Sud, France, $298 \mathrm{p}$.

2. CHESSEL D., DUfOUR A.B., THIOULOUSE J., 2004. The ade4 package-I: One-table methods. $R$ News, 4: 5-10.

3. CHESSEL D., MERCIER P., 1993. Couplage de triplets statistiques et liaisons espèces-environnement. In : Lebreton J.D., Asselain B. éds, Biométrie et environnement. Paris, France, Masson, p. 15-44.

4. CHOISIS J.P., LACROIX S., LATCHIMY J.Y., LEGENDRE E., 2003. Produire des références pour connaître et pérenniser les exploitations bovines allaitantes à la Réunion. In : Grimaud P. éd., Symp. "Les ruminants, élevage et valorisation », St-Denis, la Réunion, 10-13 juin 2003. Montpellier, France, Cirad, p. 29.

5. DARRE J.P., 1986. La production de connaissance dans les groupes locaux d'agriculteurs. Agriscope, 7 :24-35.

6. DOLEDEC S., CHESSEL D., 1994. Co-inertia analysis: an alternative method for studying species-environment relationships. Freshwater Biol., 31: $277-294$

7. DRAY S., DUFOUR A.B., CHESSEL D., 2007. The ade4 package-II: Two-table and K-table Methods. R News, 7: 47-52.

8. DURU M., 2000. Le volume d'herbe disponible par vache : un indicateur synthétique pour évaluer et conduire un pâturage tournant. Prod. Anim., 13 : 325-336.

9. DURU M., BERGEZ J.E., DELABY L., JUSTES E., THEAU J.P., VIEGAS J., 2007. A spreadsheet model for developing field indicators and grazing management tools to meet environmental and production targets for dairy farms. J. Environ. Manage., 82: 207-220.

10. GERBAUD S., 2004. Etude d'un processus de construction et d'appropriation d'outils : le cas du programme de gestion raisonnée des prairies à la Réunion. Mémoire DAA, INA Paris-Grignon, France, 80 p.

11. GRIMAUD P., COIGNAC O., MOULIN C., 2001. L'analyse fonctionnelle des systèmes d'alimentation dans les élevages bovins réunionnais sur pâturage. Rencontres Rech. Ruminants, 8 : 224. 
12. HOSTIOU N., TOURRAND J.F., HUGUENIN J., LECOMTE P., 2006. La diversité de gestion des systèmes herbagers en Amazonie. Cas des élevages bovins brésiliens. Fourrages, 187 : 377-392.

13. INSTITUT DE L'ELEVAGE, 2005. Les systèmes bovins viande en France. Repères techniques et économiques. Paris, France, Institut de l'élevage, 20 p.

14. LACROIX S., 2002. Etude du fonctionnement des exploitations naisseurs à la Réunion. Mémoire, Istom, Cergy-Pontoise, France, 51 p.

15. R DEVELOPMENT CORE TEAM, 2007. R: A Language and environment for statistical computing. Vienna, Austria, R Foundation for Statistical Computing, http://www.R-project.org

16. ROBERT P., ESCOUFIER Y., 1976. A unifying tool for linear multivariate statistical methods: the RV coefficient. J. appl. Stat., 25: 257-265.

17. ROMESBURG H.C., 1985. Exploring, confirming and randomization tests. Comput. Geosci., 11: 19-37.
18. SARZEAUD P., 2002. Coût de production et prix de revient en viande bovine - applications, résultats et limites méthodologiques. Rencontres Rech. Ruminants, 9 : 181-184.

19. SIMIER M., BLANC L., PELLEGRIN F., NANDRIS D., 1999. Approche simultanée de $\mathrm{K}$ couples de tableaux : application à l'étude des relations pathologie végétale - environnement. Revue Stat. appl. 47 : 31-46.

20. THIOULOUSE J., CHESSEL D., DOLEDEC S., OLIVIER J.M., 1996. Ade-4: a multivariate analysis and graphical display software. Stat. Comput., 7: 75-83.

21. THOMAS P., GRIMAUD P., 2002. La gestion raisonnée des prairies sur l'île de la Réunion. In : Sémin. Inra / Cirad "Les outils d'aide à la gestion des fourrages », St Pierre, la Réunion, 13-17 mai 2002.

22. VIVIER M., 1990. Les prairies et les pratiques d'exploitation. Eléments et réflexions pour un diagnostic. Fourrages, $124: 337-355$.

Reçu le 18.01.2008, accepté le 11.09.2008

\section{Summary}

Choisis J.P., Lassalle C., Messad S., Grimaud P. Animal Production Performance and Herd Management in Suckling Farms on Réunion Island

In Réunion, because of the insularity and the small size of farms, improving cattle farm productivity involves increasing technical management aspects. To analyze relationships between herd management practices and animal performances, a survey was conducted in ten suckling farms, located in the Highlands, from 1999 to 2002. Three sets of 4, 8 and 3 variables, respectively, were thus extracted from the monitoring database: animal performances (calving interval, fertility rate, body weight at standard age, live meat production), farmers' practices (grazing time per hectare and paddock, time interval between two passages, paddock size, stocking rate, feed complementation of weaned animals and lactating cows, culling rate), and environment (rainfall, herbage production, body condition score of cows). An analysis of co-inertia was carried out on the first two tables to analyze relationships between animal production performances and practices. A significant correlation was observed between the two tables. The results of the co-inertia analysis were interpreted for each farm. Beyond specific constraints, they revealed proximities between farms and herd management based on various strategies, which were relevant with the observed performances. A STATICO analysis was performed to assess relationships between performance parameters and environment parameters for the four studied years. It revealed that there was a stable costructure between the environment and performance tables. This suggests that practices had a highly structuring effect on animal production and that some system adjustments mitigated the climate effects.

Keywords: Suckler cattle - Livestock management - Animal performance - Multivariate analysis - Réunion.

\section{Resumen}

Choisis J.P., Lassalle C., Messad S., Grimaud P. Rendimientos zootécnicos y prácticas de los criadores bovinos de carne en la Reunión

En la Reunión, la insularidad y la pequeña dimensión de las explotaciones hacen que el mejoramiento de la productividad en los criaderos bovinos pase por un aumento del dominio técnico. Con el fin de analizar las relaciones entre las prácticas de conducta y los rendimientos animales, se siguieron, entre 1999 y 2002, 10 criaderos de nacimiento bovinos, situados en las Alturas de la Reunión. Los datos recolectados se organizaron en tres cuadros compuestos, respectivamente, de 4, 8 y 3 variables: los rendimientos animales (intervalo entre partos, tasa de partos, peso a edad tipo, producción bovina de carne viva), las prácticas (tiempo de presencia por hectárea y por parcela, tiempo inter pasos, tamaño de las parcelas, carga, complemento de los becerros y de las vacas, tasa de reforma) y el medio (pluviometría, volumen de hierba, estado corporal de las vacas). Los dos primeros cuadros se sometieron a un análisis de co inercia con el fin de analizar las relaciones entre los rendimientos zootécnicos y las prácticas de los criadores. Los dos cuadros estaban correlacionados significativamente y los resultados del análisis de co inercia se interpretaron a la escala de cada explotación. Más allá de los obstáculos específicos, los cuadros revelaron similitudes entre establecimientos y modos de conducta, basados sobre estrategias variadas, en coherencia con los rendimientos observados. Se realizó un análisis Statico con el fin de evaluar la relación entre los parámetros de rendimiento y los del medio para los cuatro años estudiados. Este demostró la existencia de una co estructura estable entre los cuadros de medio y rendimiento, sugiriendo un efecto estructurante de las prácticas sobre los rendimientos y la existencia de regulaciones del sistema que moderan los efectos del clima.

Palabras clave: Ganado bovino - Vaca de cría - Manejo del ganado - Desempeño animal - Análisis multivariante Reunión. 